\section{Improving the quality of health services in developing countries: lessons for the West}

\section{$\mathrm{J}$ Øvretveit}

\section{The West can learn from the experiences of developing countries on improving quality and safety.}

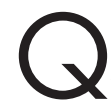

uality methods used in health care have been developed in Western health systems. Here there is a growing awareness of the waste and risks caused by problems rooted in systems of care which are not well organised. Governments and others are making resources available to address these problems, and this is being seen as a necessary investment to save money and unnecessary patient suffering. In contrast, in lower income countries the development and quality of health services is severely limited by lack of resources and knowledge about quality methods.

Despite these differences, however, lower income countries increasingly recognise the value of quality methods and the need to raise the quality of their services. Many are making more use of quality methods, but the traffic is not one way-the West can also learn from their experiences of improving quality and safety. It is worth remembering that quality methods were first developed and put into widespread use in Japan after the Second World War-a country with few resources-and then reimported into the West. This editorial considers some of the challenges in applying and adapting quality methods in these countries, as well as the potential for testing and developing more cost effective methods, some of which may be valuable for Western health care.

There are severe limitations to health care in most developing countries. One perhaps extreme example from a current programme in a low income Arabic country is presented here. The average spend on public health care per head of population is $\$ 6$ a year, and it is falling every year. Although there are many health facilities, the services are unevenly distributed and there is a lack of many essential drugs (despite various programmes to solve this problem) and inappropriate prescription. Health personnel are undertrained, unsupervised, and morale and incomes are low (about $\$ 10$ a month for doctors). Patients make little use of the public health system: mother and child services.

co-payments have been introduced but quality has not improved. An unregulated private sector is fast expanding with a large pharmaceuticals market. Poor diagnosis and inappropriate treatments waste many resources and cause unnecessary suffering and mortality. One district hospital is typical: an occupancy rate of $12 \%, 140$ staff (45 doctors seven of whom are from overseas and cannot speak the language), and diagnostics and treatments which are often ineffective or unsafe. Add to this the situation for women where the average fertility rate is 6.8 children per woman, there are few women doctors, and few

\section{"Many [developing countries] are making more use of quality methods, but the traffic is not one way..."}

Surely the issue here is how to improve the performance of the health system and how to establish a basic infrastructure which includes training and management capacity building? In a situation like this, some take the view that quality methods and concepts are irrelevant. There is certainly a case for arguing that some quality approaches are inappropriate-for example, large amounts spent on accreditation systems to improve the quality of tertiary hospital services could be put to better use. Accreditation is certainly more easy to understand than many other quality methods and it is often supported by donors, but it is often unsustainable, ineffective and inappropriate in many of these countries. In my experience, quality methods have an important part to play in improving the performance of the health system if the right ones are chosen for the situation and adapted in a culturally appropriate way. If the money used for the accreditation system was invested in quality methods to improve immunisation programmes or drug supply logistics, many more lives would be saved and the changes would be sustainable.
Even though quality methods might seem a luxury, the country in the example-in common with an increasing number of developing countrieshas introduced a national health quality strategy. Is this political "window dressing" or a response to aid donor's concerns? To some extent it is both, but also more. Highlighting quality as a problem and saying that something needs to be done is a popular move but of little use unless a low cost way can be found to get results. At the same time there is a genuine determination among some managers and practitioners to do something about the quality of services, and also a belief that quality methods might have something to offer, if only because they come from a part of the world that has brought mobile telephones and cars which work.

As one of a number of quality experts working in developing countries, I have been challenged to propose appropriate strategies. In Arabic culture, with different management traditions, I have been forced to recognise how much quality methods presuppose an attitude, way of working, and certain management processes. The relation between the Ministry of Health and local districts, ruled by tribal leaders, is more one of negotiation than direction. Introducing quality systems also means introducing management processes which challenge the existing power structures and culture and are quickly rejected. In many such countries, but not all, multidisciplinary improvement teams do not work because traditional authority structures or team approaches have to be adapted for the culture-some might say this is also true of the West.

To make progress it is necessary to find able managers and practitioners, show them the different approaches, and work with them to adapt and test what they think might work. As in the West, there are many who argue that an expansion of the existing system is all that is necessary: more doctors and nurses doing what they do now. Others argue that reallocation of resources is the way: more personnel in primary care and fewer in secondary and tertiary care. Others propose that stronger management and decentralisation is necessary to improve quality. And there are a few who propose using quality methods specifically to address problems in how care is provided and in support services. As in the West, there is debate about how many resources should be diverted into quality activities and a growing recognition that this will be a permanent extra resource demand. The choices are starker and the need for the investment to "pay off" is greater.

Although at first not knowing where to start, Ministry and local managers decided on an interesting mix of approaches. One is to define a "standard 
package of services" for health centres and units across the country-this definition does not itself bring such services to all areas, but it is a step in that direction. They rejected certification, stronger licensing, and accreditation at this stage, and decided to trial a quality management system in selected districts. Past experience has been that large numbers of standards have been developed but not followed. The trials have chosen a few health conditions where following standard procedures would make care more effective-for example, the diagnosis and treatment of acute respiratory tract infection. The emphasis is on training, supportive supervision, and action using problem solving methods to ensure these few standards are followed, then to add more standards.

One reason for choosing this approach is that it is a small achievable step which may help with the problem of motivation and incentives for health workers. Workers are finding that more effective care means more patients and higher incomes. Supervisors are more welcome and are being questioned about correct procedures for other treatments. Other approaches are being used to upgrade clinical skills and to address problems of morale and discipline. There are lessons for the West in the careful way in which policy makers with few resources analysed which issues were amenable to action and which issues were too large to take on in the early stages of the programme. There are also lessons from the way in which the donor who contributed to this programme helped address the sustainability issue: how successful projects could be transferred into routine operations with the resources to support continued quality activities.

This approach is not typical of all developing countries, but what is typical is the way in which colleagues in these countries are adapting and experimenting with quality methods. They are increasingly sceptical of "quality packages" from the West which are said to cure the ills of their health systems. Certainly many quality methods transferred from the West are unaffordable or unsustainable, and many do not translate. This raises the question of whether there is an equivalent to Maslow's "hierarchy of needs" for quality methodswhere certain steps need to be taken first before using the methods of more sophisticated continuous quality improvement or other approaches? Or are the new approaches being developed suited to the different cultures and limited resources, but could also be effective in the West?

Quality methods are severely tested in developing countries and, as in the West, many do not work in certain cultural and economic circumstances. However, the need to improve quality in these countries is as pressing, if not more so, than in the West. Some able colleagues are adapting and pioneering new methods and new approaches to using these methods, from which the West can learn. It is to be hoped that more reports of these programmes and experiments could be published in international quality journals. Dialogue and debate about effective methods in different circumstances would be of benefit, especially to patients in these countries and, in the long term, to patients in the West.

Qual Saf Health Care 2002;11:301-302

\section{Authors' affiliations}

J Øvretveit, Director of Research, The Karolinska Institute Medical Management Centre, Stockholm, and Professor of Health Policy and Management, The Nordic School of Public Health, Gothenberg, and Bergen University Faculty of Medicine, Norway

Correspondence to: Dr J Øvretveit, The Nordic School of Public Health, Gothenberg, Box 12133, Sweden S-40242; jovret@aol.com

\section{From patients to politicians: a cognitive engineering view of patient safety}

\section{K J Vicente}

\section{Sizeable and long lasting reductions in adverse events cannot be realized unless decision makers at all levels pay attention to the global system phenomenon of inadvertent harm to patients.}

M any healthcare providers now know that patient safety poses a significant risk to public health. The American statistics in particular are frequently cited: preventable medical error is the eighth leading cause of death, it is responsible for 44 000-98 000 deaths annually in hospitals alone, and it results in patient injuries that cost between $\$ 17$ billion and $\$ 29$ billion annually. ${ }^{1}$

Virtually all of the medical experts who have written on this topic have stated that the key to improving patient safety is to apply system design principles from human factors engineering. ${ }^{12}$ This discipline aims to tailor the design of technology to conform to human nature rather than expect people to contort and adapt to technology. By doing so, systems become easier for people to work in, ultimately reducing error. Human factors techniques have been applied to other industries, such as nuclear power and aviation, and have been very successful in reducing error and improving safety in these contexts.

If the magnitude of the problem is significant and widely known, and if there is a consensus on the likely remedy, then why has not more progress been made on improving patient safety? One possibility is that human factors engineering has traditionally been primarily concerned with "knobs and dials" or "graphical user interface (GUI)" interventions to improve the usability of equipment and software. Clearly, this narrow focus does not address most of the threats to patient safety; as a result, many healthcare providers see patient safety and human factors engineering as specialist concerns that are outside their sphere of action and responsibility. This attitude is a legitimate response to the narrow traditional approach to human factors, but it does not apply to cognitive engineering-a newer cognate discipline that has evolved to address the limitations of the traditional approach. ${ }^{3}$

A state of the art cognitive engineering approach begins with a much broader systems perspective, identifying the various actors-individuals, computer systems, and organizations-in a complex sociotechnical meta-system. ${ }^{45}$ Figure 1 provides a representative example, although the precise number of levels and their labels can vary across industries. In the context of health care, this hierarchy would include, from bottom to top: patients, providers, department managers, hospital CEOs and CFOs, professional regulators and associations, and government (that is, civil servants and politicians). Knowingly or not, each of these individuals and stakeholders makes decisions that affect patient safety. 


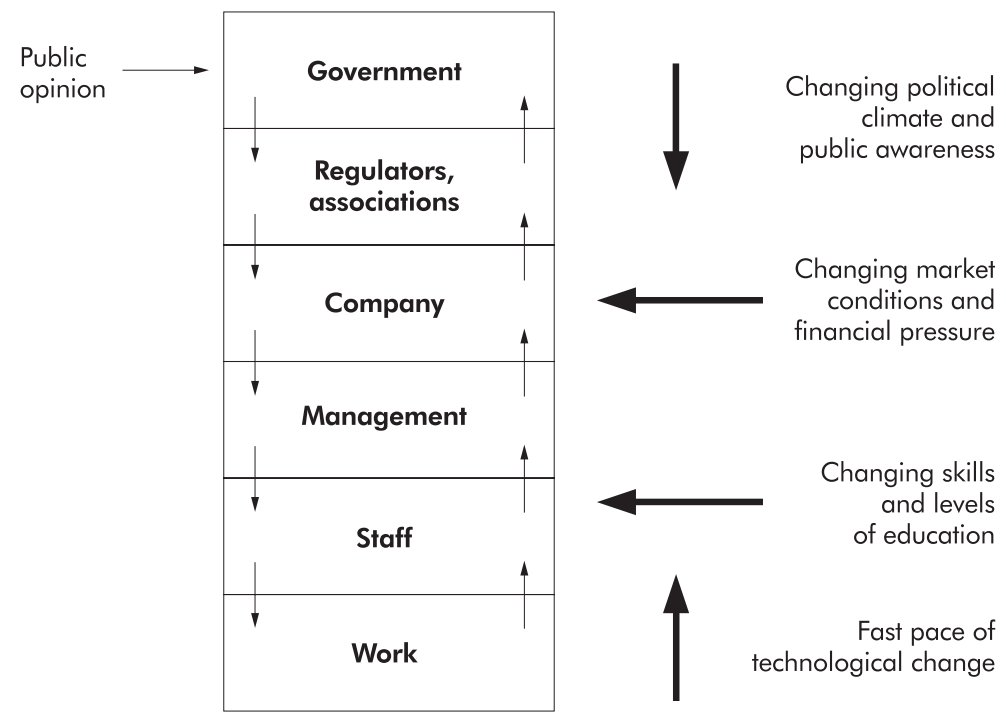

Figure 1 Various levels of a complex sociotechnical system involved in risk management. Adapted from Rasmussen. ${ }^{4}$

The growing literature on patient safety has started to address each of these levels. At the level of the individual worker, our work at the University of Toronto has shown how medical devices could be designed to make them easier for providers to use, thereby reducing errors that have been described in reports of patient deaths. ${ }^{6}$ This is the traditional realm of human factors engineering. At the management level, researchers at Stanford University have developed training programs that help anesthesiologists to manage team communication and coordination, thereby complementing more traditional training programs that focus on individual medical skills. ${ }^{7}$ At the organizational level, the US Veterans Administration has pioneered a radically different risk management policy that has led to a more humane health care environment in addition to reducing legal costs. ${ }^{8}$ Finally, at the government level, a researcher at Pepperdine University has documented how some aspects of the US legal system provide impediments to improving patient safety, thereby pointing the way towards reform. ${ }^{9}$

"Horizontal" research at each of these levels is necessary to improve patient safety. Cognitive engineering does not, and cannot, take the place of these multidisciplinary safety initiatives but, because of its broad systems view, it points to a critical factor that is overlooked by all horizontal research efforts-the additional need for "vertical" integration across the levels in fig 1 . Decisions at higher levels should propagate down the hierarchy, whereas information about the current state of affairs should propagate up the hierarchy. These interdependencies across levels of the hierarchy are critical to the successful functioning of a healthcare system as a

whole. Even if researchers do an excellent job at conducting horizontal research on a particular topic, they may have little impact on patient safety unless vertical integration is also achieved. For example, the Stanford team has conducted pioneering research on training crisis resource management skills but, unlike aviation, this type of training is not yet legally mandated in health care. Because of this mismatch between the management level and the regulatory and government levels in fig 1 , research at the management level has not had as much impact on patient safety as it can or should. Many other examples of lack of vertical integration in healthcare systems could be cited Given the available evidence from other safety-critical industries, there are strong reasons to believe that these mismatches are the most significant contributors to adverse events. ${ }^{40}$ It may be the lack of coordination across levels, not the individual levels themselves, that poses the greatest threat to patient safety.

\section{"Patient safety is everyone's business . . . from patients to politicians"}

Unfortunately, the holy grail of vertical integration is becoming more important yet more difficult to achieve. As shown on the right of fig 1 , the various layers of a complex sociotechnical system are increasingly subjected to external forces that stress healthcare systems. Examples of such perturbations are:

- changing political climate and public awareness;

- changing market conditions and financial pressures;
- changing competencies and levels of education; and

- changes in technological complexity.

In today's dynamic society, these external forces are stronger and change more frequently than ever before. When different levels of the system are being subjected to different pressures, each operating at different time scales, it is imperative that efforts to improve patient safety within a level be coordinated with the changing constraints imposed by other levels. To take a simple example, if hospital managers decide to reduce nursing staff levels to cope with budget cuts passed on from above, then the mental workload experienced by individual nurses will increase, making it even more important that medical devices be designed to minimize mental effort. Without coordinating the changes at various levels of healthcare systems, the external forces acting on the system may unintentionally be "preparing the stage for an accident". ${ }^{4}$

Patient safety is-and will continue to be-everyone's business, all the way from patients to politicians. Sizeable and long lasting reductions in adverse events cannot be realized unless decision makers at all levels pay attention, not just to their immediate local concerns, but also to the global system phenomenon of inadvertent harm to patients. The rationale behind this fundamental lesson from cognitive engineering can be revealed by a simple rhetorical acid test: if all you have are (patient) safety departments and specialists, then what does that say about all of your other departments and specialists?

\section{ACKNOWLEDGEMENTS}

The writing of this paper was sponsored in part by the Jerome Clarke Hunsaker Distinguished Professorship at MIT and by a research grant from the Natural Sciences and Engineering Research Council of Canada.

Qual Saf Health Care 2002;11:302-304

\section{Author's affiliation}

K J Vicente, Department of Aeronautics and Astronautics, Massachusetts Institute of Technology, Cognitive Engineering Laboratory, Department of Mechanical \& Industrial Engineering, Institute of Biomaterials and Biomedical Engineering, Department of Computer Science, Department of Electrical \& Computer Engineering, University of Toronto, Toronto, Ontario M5S 3G8, Canada; vicente@mie.utoronto.ca

\section{REFERENCES}

1 Kohn LT, Corrigan JM, Donaldson MS. To err is human: building a safer health system. Washington, DC: National Academy Press. 1999

2 Leape LL. Error in medicine. JAMA 1994;272:1851-7.

3 Hollnagel E, Woods DD. Cognitive systems engineering: new wine in new bottles. Int $J$ Man-Machine Systems 1983;18:583-600 
4 Rasmussen J. Risk management in a dynamic society: a modelling problem. Saf Sci 1997; 27: 183-213.

5 Vicente KJ. Cognitive work analysis: toward safe, productive, and healthy computer-based work. Mahwah, NJ: Erlbaum, 1999.

6 Lin L, Vicente KJ, Doyle DJ. Patient safety, potential adverse drug events, and medical device design: a human factors engineering approach. I Biomed Informatics 2001;34:274-84. (Available at www.idealibrary.com/links/doi/10.1006/ jbin.2001.1028/pdf, 28 March 2002).

7 Howard SK, Gaba DM, Fish KJ, et al. Anesthesia crisis resource management training: Teaching anesthesiologists to handle critical incidents. Aviat Space Environ Med 1992;63:763-70.
8 Kraman SS, Hamm G. Risk management extreme honesty may be the best policy. Ann Intern Med 1999;131:963-7.

9 Liang B. Error in medicine: legal impediments to US reform. J Health Politics Policy Law 1999;24:27-58.

10 Vicente KJ, Christoffersen K. The Walkerton E coli outbreak: a test of Rasmussen's framework for risk management in a dynamic society. Saf Sci 2002 (submitted). friendly) model which has four main sections:

(1) Introduction: 2-3 paragraphs, typically starting with a description of the topic and ending with a description of the intervention.

(2) Methods: 7-8 paragraphs describing what they did.

(3) Results: 7-8 paragraphs describing what they found.

(4) Discussion: 6-7 paragraphs starting with a summary of what they found and ending with what it all means-in other words, the message. ${ }^{1}$

This structure is not always appropriate for quality improvement articles, and in 1999 the editors decided to introduce an alternative structure for writing about local quality improvement work. ${ }^{2}$ Our advice is to choose the one with which you feel more comfortable and fits your work. Decide how many sections you will need, the number of paragraphs in each, and where the key sentences should appear. Use this as a template to construct your own brief plan.

Then write. One way is to construct a cosy nest lined with your data and references, block off 3 or 4 hours of valuable time, and painstakingly construct the article by transferring words and numbers from one piece of paper to another. A better way is to find a quiet corner, block off about 10 minutes, and "free write" each of your sections at a time without hesitation, leaving blanks where necessary. This is a creative activity and some people admit they enjoy it. This "free writing" technique ${ }^{3}$ may make you profoundly uneasy, but you will find that what your first draft lacks in details (easily inserted later) it makes up for with focus and structure.

After a sensible interval (at least overnight) to increase the chances of objectivity, you will have to rewrite. Check your facts (and check them again-there is nothing that puts off an editor so much as a column of figures that does not add up!). Insert your references: doing them at this stage will ensure that they are used for their rightful purpose, which is to back up your statements. Make sure you have a "good English style", which is harder than it sounds because your co-authors will have different views. So follow the advice of the 
recognised masters ${ }^{4-6}$ and keep your sentences short and active ("We did this" rather than "This was done"). Use short and familiar words rather than the long and pompous ("had" rather than "possessed"; "met" rather than "encountered"). Make every word count.

So far, relatively easy. Now you will need courage as you release your offspring into the wicked world of $\mathrm{CO}^{-}$ authorship and then submission. Other people will reward your efforts with countless marks in red ink. Don't be discouraged: they do this because this is what they are expected to do rather than for any failure on your part. Keep your nerve: use their comments sensibly to improve the chances of persuading the editors that this paper will be the one in three that they accept for publication. If your co-authors insist, for example, that writing must be long and pompous, show them this article. Keep focused on the main task, which is to send the article off as soon as the co-authors can agree. Don't hold out for perfection. Aim for submission, and when you achieve that aim, celebrate.

Qual Saf Health Care 2002;11:304-305

.

Conflict of interest: Tim Albert makes his living by running courses on writing and editing skills for health professionals.
Correspondence to: Tim Albert, Tim Albert Training, Dorking Surrey RH4 IQT, UK; tatraining@compuserve.com

\section{REFERENCES}

1 Albert T. Winning the publications game. Abingdon: Radcliffe Medical Press, 2000 47-51.

2 Moss F, Thomson R. A new structure for quality improvement reports. Qual Health Care 1999;8:76

3 Klauser HA. Writing on both sides of the brain. New York: Harper Collins, 1987.

4 Strunk W, White EB. The elements of style. 4th ed. Boston: Alleyn and Bacon, 2000

5 Orwell G. Politics and the English Language in the Orwell reader. New York: Harcourt Brace, 1984.

6 Amis K. The King's English. London: Harper Collins, 1997. 